\title{
Propriedades ópticas a serem considerados na seleção de cores em Odontologia estética: uma revisão de literatura
}

\author{
Optical properties to be considered in color selection in cosmetic Dentistry: a literature review \\ Propiedades ópticas a considerar en la selección del color en Odontología cosmética: revisión de la
}

literatura

Recebido: 09/01/2021 | Revisado: 11/01/2021 | Aceito: 13/01/2021 | Publicado: 17/01/2021

Erika Thaís Cruz da Silva

ORCID: https://orcid.org/0000-0002-8475-7929

Universidade Estadual da Paraíba, Brasil

E-mail: erika-thais-silva@ hotmail.com

Adriano Flores da Silva

ORCID: https://orcid.org/0000-0001-7515-4088 Universidade Estadual da Paraíba, Brasil

E-mail: floresuepb@gmail.com

Suellen Rabelo Rocha da Costa

ORCID: https://orcid.org/0000-0003-0841-2061

Universidade Estadual da Paraíba, Brasil E-mail: suellenrabelo@ hotmail.com.br

Priscila Lima Bezerra

ORCID: https://orcid.org/0000-0003-2458-498X Universidade Estadual da Paraíba, Brasil

E-mail: eupriscilalimabe@gmail.com

Aretha Helen Aragão Lourenço

ORCID: https://orcid.org/0000-0001-8851-3473 Universidade Estadual da Paraíba, Brasil

E-mail: arethaaragao6@gmail.com

Nilson Emmanuel Gomes Pereira

ORCID: https://orcid.org/0000-0002-4974-7119

Universidade Estadual da Paraíba, Brasil

E-mail: nilsonegp@gmail.com

\begin{abstract}
Resumo
O objetivo do presente trabalho foi realizar uma revisão de literatura acerca das propriedades ópticas e sua influência na seleção de cores em odontologia estética. Foi realizada uma revisão de estudos disponíveis na literatura por meio da busca bibliográfica nas bases de dados eletrônicos Pubmed/Medline, Lilacs, Science direct e Scielo (scientific eletronic library). para a pesquisa foram utilizados os seguintes descritores: "propriedades ópticas", "odontologia estética", "seleção de cores" e "fluorescência". Os artigos foram selecionados obedecendo-se aos critérios de inclusão e exclusão do estudo. Também foram adicionados alguns livros com conteúdos relevantes para esta pesquisa. Assim como, foi indispensável à disponibilidade do texto integral para sua inclusão no estudo. A escolha correta da cor é o processo que representa uma das maiores dificuldades dos profissionais, pois não depende apenas da técnica e da experiência profissional, mas também de uma série de fatores relacionados ao meio em que estão inseridos os dentes. As propriedades ópticas apresentam grande relevância na seleção de cores em procedimentos estéticos na odontologia. É importante que o profissional conheça essas propriedades e aplique esses conhecimentos na prática clínica diária para que obtenha os melhores resultados possíveis em seus casos.
\end{abstract}

Palavras-chave: Propriedades ópticas; Odontologia estética; Fluorescência.

\begin{abstract}
The aim of this study was to perform a literature review about optical properties and their influence on color selection in cosmetic dentistry. A review of studies available in the literature was performed through bibliographic search in the electronic databases Pubmed / Medline, Lilacs, Science direct and Scielo (scientific electronic library). the following descriptors were used for the research: "optical properties", "aesthetic dentistry", "color selection" and "fluorescence". The articles were selected according to the inclusion and exclusion criteria of the study. Some books with relevant content for this research were also added. As well, it was essential to the availability of the full text for inclusion in the study. The correct choice of color is the process that represents one of the greatest difficulties for professionals, as it depends not only on technique and professional experience, but also on a series of factors related to the environment in which the teeth are inserted. Optical properties are highly relevant in the selection of colors in
\end{abstract}


cosmetic procedures in dentistry. It is important that the professional knows these properties and applies this knowledge in daily clinical practice to obtain the best possible results in their cases.

Keywords: Optical properties; Aesthetic dentistry; Fluorescence.

\section{Resumen}

El objetivo de este estudio fue realizar una revisión de la literatura sobre las propiedades ópticas y su influencia en la selección del color en la odontología cosmética. Se realizó una revisión de los estudios disponibles en la literatura mediante búsqueda bibliográfica en las bases de datos electrónicas Pubmed / Medline, Lilacs, Science direct y Scielo (biblioteca electrónica científica). Para la investigación se utilizaron los siguientes descriptores: "propiedades ópticas", "odontología estética", "selección de color" y "fluorescencia". Los artículos fueron seleccionados según los criterios de inclusión y exclusión del estudio. También se agregaron algunos libros con contenido relevante para esta investigación. Además, era fundamental que el texto completo estuviera disponible para su inclusión en el estudio. La correcta elección del color es el proceso que representa una de las mayores dificultades para los profesionales, ya que depende no solo de la técnica y experiencia profesional, sino también de una serie de factores relacionados con el entorno en el que se insertan los dientes. Las propiedades ópticas son muy relevantes en la selección de colores en procedimientos cosméticos en odontología. Es importante que el profesional conozca estas propiedades y aplique estos conocimientos en la práctica clínica diaria para obtener los mejores resultados posibles en sus casos.

Palabras clave: Propiedades ópticas; Odontología estética; Fluorescencia.

\section{Introdução}

Um dos maiores desafios da odontologia restauradora estética é confeccionar restaurações que estejam o mais próximo possível dos dentes naturais e sejam difíceis de serem detectadas pelas pessoas que o observem. No entanto, é importante ressaltar que a estética é algo que pode variar de acordo com a percepção de cada pessoa ou profissional (Darvel, 2012; Busato, 2005).

A forma, função e estética dos dentes são características bastante importantes em odontologia estética. Os fenômenos ópticos possuem grande relevância nesse contexto, principalmente aqueles que se referem a propagação da luz e da cor. Ao longo do tempo, com a modernização dos materiais, os aspectos estéticos em odontologia restauradora e protética passaram a ser bastante relevantes. Isso acontece porque mesmo com o avanço da tecnologia na fabricação dos materiais dentários, ainda existem muitos desafios a serem superados quanto ao desenvolvimento de um material restaurador pouco sensível à técnica da seleção de cor do dente e estabilidade no que concerne a manutenção da cor. Sabe-se que o desgaste do esmalte, da deposição fisiológica de dentina e à pigmentação por corantes são fatores que levam a mudança de cor dos dentes. Portanto, é de suma importância que os profissionais conheçam esses aspectos ópticos que influenciam na seleção de cores para que cheguem ao máximo de excelência possível (Reis et al., 2007; Phillips, 2013).

Para identificar as características visuais dos tecidos dentários que compõem a aparência geral dos dentes, realizamos um processo chamado “seleção de cor”. Porém, a cor é apenas uma das várias dimensões que influenciam na aparência geral dos objetos, as quais são denominadas propriedades ópticas, sendo elas: forma, textura, opacidade, translucidez, opalescência, fluorescência, brilho e a cor propriamente dita, subdividida em três dimensões (valor, matiz e croma) (Salgado et al., 2019).

A percepção de determinada cor acontece através de uma resposta do cérebro a algum estímulo luminoso e, portanto, a cor depende da existência de um observador, da luz e do objeto. Determinar a cor em restaurações, sejam elas diretas ou indiretas, pode ser bastante desafiador para o cirurgião-dentista tendo em vista que, a assimilação da cor representa uma resposta fisiológica a um estímulo físico, sendo portanto algo bastante subjetivo. Outros aspectos importantes na determinação da cor são as condições de iluminação e a fonte de luz, que devem ser adequadas para que se alcançe o melhor resultado possível. (Noort, 2010; Santos, 2014; Salgado et al., 2013).

Diante do exposto acima, o presente trabalho tem como objetivo realizar uma revisão de literatura acerca das propriedades ópticas e sua influência na seleção de cores em odontologia estética. 


\section{Metodologia}

Foi realizada uma revisão de estudos disponíveis na literatura por meio da busca bibliográfica nas bases de dados eletrônicos Pubmed/Medline, Lilacs, Science direct e Scielo (scientific eletronic library). para a pesquisa foram utilizados os seguintes descritores: "propriedades ópticas", "odontologia estética", "seleção de cores" e "fluorescência". Outra estratégia de pesquisa foi à busca manual em listas de referências dos artigos selecionados. Também foram adicionados alguns livros com conteúdos relevantes para esta pesquisa. Como critérios de inclusão, foram adotados os artigos escritos em inglês, espanhol e português; aqueles que se enquadravam no enfoque do trabalho e os mais relevantes em termos de delineamento das informações desejadas.

Foram observados e determinados alguns aspectos para a inclusão dos estudos na revisão; como a significância, a confiabilidade e clareza no detalhamento metodológico das informações apresentadas. Assim como, foi indispensável à disponibilidade do texto integral para sua inclusão no estudo. Foram excluídos da amostra os artigos que não apresentaram relevância sobre o tema abordado e aqueles que não se enquadraram nos critérios de inclusão.

\section{Revisão de Literatura}

A forma como a luz é transmitida, absorvida ou refletida influencia diretamente nas cores que são vistas por nossos olhos. A luz incidente é conhecida como "luz branca" é normalmente policromática e representa uma mistura de diversos comprimentos de onda. A luz que atravessa e o comprimento de onda absorvido é determinado pela espessura, superfície e densidade do material. Se toda a luz for absorvida, não será possível observar nenhuma cor, resultando no negro (preto). Contudo, se toda luz for refletida, será possível observar a cor branca. Sendo assim, se um objeto possuir a cor branca, ele possui a capacidade de dispersar de forma difusa e uniforme toda radiação incidente, ou seja, sem nenhuma absorção. Se uma luz branca incide sobre um objeto e conseguimos observar a cor verde, isso significa dizer que apenas os comprimentos de onda interpretados como essa cor foram refletidos e todos os outros comprimentos de onda do espectro visível foram absorvidos (Phillips, 2013; Chu et al., 2012).

As propriedades ópticas influenciam também na cor das resinas compostas, e características como composição, tamanho, quantidade e distribuição espectral das partículas de carga presentes no material podem influenciar na cor dessas resinas. ${ }^{38}$.

É importante compreender que existem três medidas fundamentais para descrever as cores. Elas são chamadas de três dimensões das cores de modo que a combinação das dimensões das cores nos fornece a compreensão da tonalidade da cor (Dallanora et al., 2014; Lucena et al., 2016).

Ao observarmos a cor dos dentes podemos perceber que eles apresentam grande variação de cores, alternando-se entre entre tons de amarelo e laranja, dessa forma, não é fácil descrever a aparência e cor dos dentes apenas com simples descrições. Um artista americano chamada A.H. Munsell criou um método no ano de 1995 que classifica as cores de acordo com três dimensões que são denominadas de croma, matiz e valor. Esse método é aceito internacionalmente e possibilita uma melhor compreensão das cores tornando possível quantificar, transmitir e reproduzir uma determinada cor com bastante precisão (Souza Junior, 2000).

O matiz representa a cor dominante, ou seja, se você diz que uma cor é azul, verde ou amarelo, está se referindo ao seu matiz. Em dentes naturais, a dentina é o principal responsável pelo matiz básico do dente. Já o croma, representa a intensidade ou saturação de determinada cor, ou seja, de determinado matiz. Por exemplo, o vermelho pode variar desde o “escarlate" que tem uma alta saturação, até ao rosa-claro que possui uma baixa saturação. O valor representa a quantidade de luz refletida por um objeto. Em odontologia, é a medida mais importante de ser observada, de forma que é através do valor que 
será possível a distinção entre cores claras e escuras. Essa distinção é imprescindível para que se adeque às características específicas de brilho do dente em cada paciente (Phillips, 2013; Joiner, 2004).

Outro fenômeno óptico importante que também deve ser observado em restaurações com resina composta é a translucidez. Ela é considerada como uma quarta dimensão fundamental na determinação das cores em odontologia. A utilização de resinas translúcidas em dentes anteriores possui o objetivo de simular o esmalte dentário em todas as faces (vestibular, palatina, proximais e incisais). A translucidez pode levar a falhas e resultados estéticos bastante negativos caso a translucidez desses tratamentos restauradores não busque mimetizar o máximo possível a estrutura dental natural. Resinas para corpo, dentina e esmalte devem ser colocadas em espessuras adequadas para obtenção de níveis corretos de opacidade e translucidez, até mesmo em situações que a escolha do matiz, croma e valor estejam corretos) (Silva et al., 2016; Santos, 2014).

Podem ser observadas três níveis de translucidez nas resinas compostas. Nas resinas incisais indicadas para restaurações do esmalte de margens incisais altamente translúcidas observadas em alguns dentes pode ser observada uma alta translucidez. A média translucidez pode ser observada nas resinas de esmalte que são indicadas para a restauração do esmalte da maior parte dos dentes, sendo também denominadas por alguns fabricantes como resina de corpo. E por último, a baixa translucidez que pode ser encontrada nas resinas para dentina utilizadas para restaurar o tecido dentinário perdido, sendo denominadas por alguns fabricantes de resina opaca (Chain, 2013).

Os dentes são estruturas policromáticas, ou seja, podem ser observadas diversas sensações de cores de acordo com diferentes situações e também em diferentes regiões dos dentes. Por esse motivo, selecionar corretamente a cor de um dente pode ser um processo bastante desafiador. Além disso, diversas propriedades ópticas podem ser observadas em sua composição estrutural (esmalte, dentina e polpa) concomitantemente com as variadas cores e espessuras de esmalte e dentina observadas ao longo do dente, bem como, da forma como se distribuem pela coroa dentária (Ribeiro, 2009; Baltzer \& Jinoian, 2004).

Existem objetos que são capazes de absorver luz de um comprimento de onda próximo à faixa ultravioleta (300$400 \mathrm{~nm}$ ) e posteriormente a liberam como uma luz de comprimento de onda maior que a radiação incidente (400-450nm). Essa propriedade é denominada fluorescência e em dentes naturais é caracterizada pela absorção de luz ultravioleta ou "luz negra" gerada, por exemplo, por lâmpadas presentes em boates e casas noturnas, que é invisível aos nossos olhos e, em seguida, ocorre a emissão de luz visível com comprimento de onda maior. A interpretação da cor é normalmente como mais branca e azulada. Esse fenômeno ocorre de forma natural três vezes mais na dentina do que no esmalte devido ao maior conteúdo de moléculas orgânicas fotossensíveis como a piridinolina, a timina e o triptofano presentes na dentina intertubular. O motivo pelo qual os dentes aparecem mais brancos sob uma luz fluorescente e também o porquê é mais fácil distinguir coroas, pontes ou restaurações sob uma mesma luz fluorescente do que sob a luz do dia é devido a esta propriedade óptica (Chu et al., 2012; Torres et al., 2013; Santos, 2014).

Caso a restauração não possua a fluorescência adequada, isso pode resultar em uma aparência estética desagradável em locais que possuam luz negra, de modo que as restaurações podem tornar-se perceptíveis com áreas escuras. Na tentativa de simular ao máximo possível a fluorescência dental, alguns fabricantes produziram resinas compostas com maior fluorescência que os dentes naturais, todavia, é preconizado o uso de compostos que simulem a fluorescência natural do dente. Os compostos responsáveis por trazer a propriedade de fluorescência às resinas estão inseridos na matriz resinosa como exemplo, o térbio e o cério (Chu et al., 2012; Santos, 2014).

Outra propriedade importante que deve ser considerada na seleção de cores em odontologia é opalescência que é uma característica inerente ao esmalte dentário. Objetos opalescentes são aqueles que possuem uma cor quando observados por luz refletida e outra, quando observados pela luz transmitida e, portanto, refletem ondas curtas e transmitem ondas longas (Lee, 2016; Torres et al., 2013; Russo, 2010; Vanini, 2001). 
Escolher a cor adequadamente é uma etapa que representa uma das maiores dificuldades dos profissionais, tendo em vista que não depende apenas da técnica e da experiência profissional, mas sobretudo de vários fatores relacionados ao meio em que estão inseridos os dentes. Em dentes anteriores a seleção de cores será mais crítica do que em dentes posteriores. Além disso, é imprescindível que esta etapa seja realizada antes do início do tratamento restaurador. Entretanto, o diagnóstico definitivo da cor pode ocorrer durante ou até mesmo após o término do tratamento (Busato, 2005; Ribeiro, 2009; Gomés-Polo, 2014).

\section{Conclusão}

As propriedades ópticas apresentam grande relevância na seleção de cores em procedimentos estéticos na odontologia. É importante que o profissional conheça essas propriedades e aplique esses conhecimentos na prática clínica diária para que obtenha os melhores resultados possíveis em seus casos. Sugere-se para trabalhos futuros sejam apresentados o surgimento de novas técnicas e tecnologias que visem melhorar a seleção de cores em odontologia estética.

\section{Referências}

Ahmad, I. (2008). Protocolos para restaurações estéticas previsíveis. Artmed.

Anusavice, K. J. (2013). Phillips materiais dentários. (12a ed.), Elsevier.

Baltzer, A., \& Jinoian, V.K. (2004). Determination of tooth colors. Quintessenz Zahntech. 30, 726-40.

Busato, L. S. (2005). Dentística - Filosofia, Conceito e Prática Clínica. Grupo Brasileiro de Professores de Dentística. Artes Médicas.

Chain, M. C. (2013). Materiais dentários. Artes Médicas. (Série Abeno: Odontologia Essencial - Parte Clínica.

Chu, S. J., Devigus, A., Paravina, R. D., \& Mieleszko, A. J. (2012). Fundamentos da Cor: Seleção e Comunicação da Cor em Odontologia Estética. (2a ed.), Editora Quintessence.

Conceição, E. N. (2007). Dentística: saúde e estética. (2a ed.), Artes Médicas Sul.

Gómes-Polo, C., Gómes-Polo, M., Vinuela, A. C., Parga, J. A. M. V. (2014). Differences between the human eye and the spectrophotometer in the shade matching of tooth colour. Journal of dentistry. 42(6): 742-745.

Joiner, A. (2004). Tooth color: a review of the literature. J Dent. 32(Suppl 1): 3-12.

Lee, Y. K. (2016). Opalescence of human teeth and dental esthetic restorative materials. Dent Mater J. 35(6), 845-854.

Ribeiro, A.H. (2009). Critérios utilizados por cirurgiões-dentistas no processo de seleção das cores de resinas compostas[Monografia]. Campinas: Centro de Pesquisas Odontológicas São Leopoldo Mandic.

Salgado, V. E., Marques, R. C., Soares, T. R. C., Cavalcante, L. M., \& Schneider, L. F. (2019). Autopercepção de cirurgiões-dentistas sobre a seleção da cor em odontologia. J Clin Dent Res. 16(2), 74-83.

Santos, A. E. C. (2014). Odontologia Integrada do Adulto. Editora Santos.

Silva, A. F., \& Lund, R. G. (2016). Dentistica Restauradora do planejamento à execução. Ed Santos.

Torres, C. R. G. et al. (2013). Odontologia Restauradora - Estética e Funcional: Princípios para a Prática Clínica. Editora Santos.

Torres, C. R. G. et al. (2013). Odontologia Restauradora - Estética e Funcional: Princípios para a Prática Clínica. Editora Santos.

Touati, B., Miara, P., \& Nathanson, D. (2000). Odontologia Estética e Restaurações Cerâmicas. Santos.

Vanini, L., \& Mangani, F. M. (2001). Determinat e communication do color using the five color dimensões de teeh. Pract Procede Aesthet Den. 13(1), 19-26. 